vessels each of which was connected to a gas burette by means of an outlet tube. The upper vessel contained the corroding medium which was in contact with the metal and the lower one contained air. The air in the headspace of the upper chamber and in the solution was replaced by nitrogen and the whole apparatus was set up in a room held at $25^{\circ} \mathrm{C}$.

Both the upper and lower burettes soon began to register an increase in volume. That in the upper one was the more rapid at first, but slackened later, and when the apparatus was taken down after two days, there was about 30 c.c. of hydrogen on either side, leaving about 9 c.c. (calculated from the loss in weight of the sheet) to be accounted for by absorption into the metal and by solution in the citric acid. I should be glad to know whether the passage of hydrogen through steel under similar conditions to the above has been recorded.

Low Temperature Research Station,

T. N. Morris.

Downing Street, Cambridge.

Jan. 12.

\section{Interaction of Radio Waves}

THE phenomenon recently reported by Tellegen ${ }^{1}$ whereby the new broadcasting station at Luxembourg appears to interact with that portion of the carrier wave of the Beromunster station which is received in Holland, can be explained by taking into account the effect of such a powerful station $(200 \mathrm{kw}$. and $\lambda=1190 \mathrm{~m}$.) on the mean velocity of agitation $(u)$ of the electrons in the ionosphere. Any change in $u$ will produce a change in $\nu$, the frequency of collision of an electron with molecules, and hence a change in the absorbing power of that part of the ionosphere in the vicinity of the station. Since this change depends on the magnitude of the electric vector in the disturbing wave, it follows that the absorbing power of this part of the ionosphere will vary in accordance with the modulation frequency of the station, and so the modulation will be impressed in part on any other carrier wave which may traverse this region.

We have examined these points quantitatively with the help of data obtained by Townsend and Tizard $^{2}$ on the motions of electrons in air, and have arrived at the following conclusions.

The amount of modulation of a carrier wave produced by a disturbing station of power $P$ and modulation frequency $f$ is approximately proportional to $P$ and inversely proportional to $f$. There is thus introduced a distortion of the original modulation, at the expense of the higher frequencies of modulation.

The variation of the impressed modulation with the wave-length of the disturbing station is more complicated, being roughly proportional to $1 /\left\{\nu^{2}+(p-\omega)^{2}\right\}$ where $p=2 \pi c / \lambda, \omega=H_{p} e / \mathrm{cm}$ and $H_{p}$ is the component of the earth's magnetic field perpendicular to the electric vector of the disturbing wave. It is clear that the quasi-resonant state $(p=\omega)$ can exist only in very localised regions of the ionosphere, and will contribute little to the total impressed modulation, which may be received over the whole path of the wave in the absorb. ing regions of the ionosphere. The disturbance will therefore be greatest when $\omega$ is small, that is, when the entire electric vector of the wave lies in the direction of the earth's magnetic field. The magneto-ionic theory shows that under European conditions this can occur only for that part of the wave's path which is roughly horizontal. In such circumstances $\omega$ will always be small for waves much longer than $214 \mathrm{~m}$.

We have examined the magnitude of the disturbance which would be experienced at Eindhoven when listening to the Beromunster station, and find that it would become appreciable for values of air pressure in the absorbing regions near those generally accepted. The disturbance experienced is proportional to $v^{2}$, so that we should expect increased disturbance at times when the sky wave is weakened by increased absorption, for example, around sunrise and sunset, and in the daytime if signals be audible.

It is to be anticipated that the Warsaw station will also exhibit the effect in just appreciable intensity if careful investigation be made. It is not to be expected, however, that the very long wave highpower telegraph stations, such as Rugby or Nauen, could produce the effect, for such long wave-lengths are probably reflected at a level in the ionosphere below that which absorbs waves of broadeasting frequencies. Neither would such a station appreciably influence the reception of other very long wave stations, since most of the received signal on these wave-lengths is due to the ground wave.

The details of our investigation will be published elsewhere in the near future, together with a dis. cussion of the possibility of utilising the phenomenon to derive further information about the ionosphere.

Department of Physics,

V. A. BaImey.

University of Sydney.

Commonwealth Radio Research Board, Sydney.

Nov. 29.

1 Nature, 131, 840, June 10, 1933.

Audibility of Auroras and Low Auroras

I WAS much interested in the article "Audibility of Aurora and Low Aurora" by F. T. Davies and B. W. Currie which appeared in Nature of December 2 , because I once witnessed an aurora and heard the swishing sounds referred to.

During the winter of 1908-1909, while attending Trinity College at Hartford, Conn., I observed a magnificent aurora. The light effects gave me the impression that the atmosphere was filled with fog, and that someone was illuminating it by playing a searchlight back and forth. The effect was very striking because the display was so close to the ground that I seemed to walk right through the illuminated fog.

The sound which I heard is exactly described by the word swishing. I do not believe I could say the swishing sound was in unison with the flickering of the lights because the sight was so new and strange that I did not observe it from the point of view of a scientist. All that I can say is the swishing sounds were heard while the lights were changing.

Research Laboratory,

General Electric Company,

1 River Road,

Schenectady, N.Y. Jan. 4. 